BRONCHIECTASIS

\title{
Inhaled fluticasone in bronchiectasis: a 12 month study
}

\author{
K W Tsang, K C Tan, P L Ho, G C Ooi, J C Ho, J Mak, G L Tipoe, C Ko, C Yan, W K Lam, \\ $M$ Chan-Yeung
}

Thorax 2005;60:239-243. doi: 10.1136/thx.2002.003236

\begin{abstract}
See end of article for authors' affiliations .....................

Correspondence to: Professor K W T Tsang, Department of Respiratory and Critical Care Medicine, University Department of Medicine, Queen Mary Hospital, Pokfulam Road, Hong Kong; kwttsang@hku.hk

Received

18 December 2002

Accepted 20 October 2004
\end{abstract}

\begin{abstract}
Background: The clinical efficacy of inhaled corticosteroid (ICS) treatment has not been evaluated in bronchiectasis, despite the presence of chronic airway inflammation.

Methods: After three consecutive weekly visits, 86 patients were randomised to receive either fluticasone $500 \mu \mathrm{g}$ twice daily ( $\mathrm{n}=43,23 \mathrm{~F}$, mean (SD) age 57.7 (14.4) years) or matched placebo $(n=43,34 \mathrm{~F}$, 59.2 (14.2) years) and reviewed regularly for 52 weeks in a double blind fashion.

Results: 35 and 38 patients in the fluticasone and placebo groups completed the study. Significantly more patients on ICS than on placebo showed improvement in 24 hour sputum volume (OR $2.5,95 \% \mathrm{Cl} 1.1$ to 6.0, $p=0.03$ ) but not in exacerbation frequency, forced expiratory volume in 1 second, forced vital capacity, or sputum purulence score. Significantly more patients with Pseudomonas aeruginosa infection receiving fluticasone showed improvement in 24 hour sputum volume (OR 13.5, 95\% Cl 1.8 to 100.2 , $p=0.03$ ) and exacerbation frequency (OR 13.3, 95\% Cl 1.8 to 100.2, $p=0.01$ ) than those given placebo. Logistic regression models revealed a significantly better response in sputum volume with fluticasone treatment than with placebo among subgroups of patients with 24 hour sputum volume $<30 \mathrm{ml}(p=0.04)$, exacerbation frequency $\leqslant 2$ /year $(p=0.04)$, and sputum purulence score $>5(p=0.03)$.

Conclusions: ICS treatment is beneficial to patients with bronchiectasis, particularly those with $P$ aerurginosa infection.
\end{abstract}

B ronchiectasis is a debilitating disease of heterogeneous aetiology, and affected patients suffer from chronic sputum production and recurrent exacerbations. The three pathogenic elements in bronchiectasis-namely, airway infection, inflammation and enzymatic activities ${ }^{12}$-interact to result in progressive airway damage. ${ }^{3}$ Many patients eventually harbour Pseudomonas aeruginosa (PA) in their airway, which is associated with significant morbidity. ${ }^{4}$

Intense and harmful neutrophilic infiltration into the bronchiectatic airway occurs, mediated by proinflammatory mediators such as interleukin (IL)-1, IL-8, tumour necrosis factor (TNF)- $\alpha$, and leukotriene (LT) B4. ${ }^{3}$ Release of neutrophil derived toxic products such as elastase and matrix metalloproteinases is upregulated in bronchiectasis, and these further damage the structure and functioning of the airway mucosa by digesting airway elastin, basement membrane collagen, and proteoglycan. ${ }^{5}$ The sputum elastase level correlates with sputum production, lung function, and airway cytokine expression in bronchiectasis. ${ }^{2}$

Systemic corticosteroid therapy reduces sputum production in patients who develop bronchiectasis secondary to the bronchiolitis obliterans syndrome, ${ }^{6}$ and improves growth and spirometric parameters but has unacceptable side effects in cystic fibrosis (CF). ${ }^{7}$ There have only been two controlled studies of the use of inhaled corticosteroid (ICS) in non-CF bronchiectasis $^{18}$ and several in CF. ${ }^{9-12}$ These suggest that ICS treatment improves bronchial hyperresponsiveness, respiratory symptoms, and spirometric parameters in $\mathrm{CF} .{ }^{10-12}$ Treatment with inhaled beclomethasone for 6 weeks reduced 24 hour sputum production by $18 \%$ and resulted in marginal improvements in peak expiratory flow rate (PEFR), forced expiratory volume in 1 second $\left(\mathrm{FEV}_{1}\right)$, and cough in 20 patients with non-CF bronchiectasis. ${ }^{8}$ We have reported that administration of inhaled fluticasone for 8 weeks improved sputum leucocyte density and proinflammatory cytokines in bronchiectasis. ${ }^{1}$ Although ICS treatment might therefore reduce leucocyte trafficking into the bronchiectatic airways and alleviate further damage, ${ }^{2}$ its efficacy in bronchiectasis has not been examined. We have undertaken a randomised, double blind, placebo controlled study to evaluate the clinical efficacy of 12 months of treatment with inhaled fluticasone in patients with stable bronchiectasis.

\section{METHODS}

\section{Patient selection}

Patients with HRCT proven bronchiectasis were recruited with written informed consent. Inclusion criteria included absence of asthma or other unstable systemic diseases ${ }^{13}$ and presence of stable bronchiectasis $(<20 \%$ alteration in 24 hour sputum volume, $\mathrm{FEV}_{1}$ and forced vital capacity (FVC) with no deterioration in respiratory symptoms at baseline visits). ${ }^{1}$ Exclusion criteria included unreliable clinic attendance, known adverse or allergic reactions to fluticasone or quinolones, and regular usage of ICS. Each patient was confirmed to be in a stable condition by three consecutive weekly assessments before randomisation (block of 4) to receive either fluticasone $(500 \mu \mathrm{g}$ twice daily) or placebo administered with the Accuhaler device. Evaluation of clinical and laboratory parameters was performed by the same team at $-2,-1,0,4,12,24,36,48$, and 52 weeks. The study protocol was approved by the institutional ethics committee.

\section{Clinical assessment}

The number of bronchiectatic lung lobes (including lingula) was determined from an HRCT scan performed within the previous 12 months for each patient. ${ }^{14}$ The number of exacerbations occurring in the preceding 12 months was determined from the history and review of clinical charts. An exacerbation was defined as persistent ( $\geqslant 24$ hour) deterioration in at least three respiratory symptoms (including cough, dyspnoea, haemoptysis, increased sputum purulence or volume, and chest pain), with or without fever $\left(\geqslant 37.5^{\circ} \mathrm{C}\right)$, radiographic deterioration, systemic disturbances, or deterioration in chest signs. ${ }^{1}$ Lung function was measured with a SensorMedics 2200 (SensorMedics, Yorba Linda, USA) 
package. A structured respiratory history and physical examination was undertaken at each visit.

\section{Sputum examination}

The volume of a 24 hour sputum collection (to the nearest $\mathrm{ml}$ ) was determined as the mean of a 3 day collection. ${ }^{1}$ Fresh sputum was obtained from each patient after chest physiotherapy. Sputum purulence was scored as $0,1,2,3,4,5,6,7$ or 8 (absence of, completely transparent, almost transparent, translucent but colourless, opaque and milky white, grey, pale green, moderately green, and dark green sputum, respectively) by the same experienced technician. ${ }^{4}$ Standard microbiological procedures were employed, using enriched and selective media, to identify all sputum bacteria. ${ }^{1}{ }^{4}$

\section{Treatment protocol for exacerbations}

Each patient telephoned the principal investigator (KWT) when there was deterioration in respiratory symptoms and was assessed within 24 hours to confirm the presence of an exacerbation. Oral levofloxacin (300 mg twice daily for 10 days) was prescribed empirically for all exacerbations. Patients were telephoned to ensure satisfactory progress. When clinical deterioration occurred, the patient would either be admitted to hospital or followed up as deemed necessary. Compliance was enforced by weekly telephone calls and diary cards, and monitored by checking the number of remaining doses displayed on the Accuhalers.

\section{Assessment of response to treatment}

A treatment response was attributed to each of the primary outcome (24 hour sputum volume, cumulative exacerbation frequency) and secondary outcome (sputum purulence score, $\mathrm{FEV}_{1} \%$, and $\mathrm{FVC} \%$ ) parameters by comparing the data following treatment with the baseline data for each patient. Improvement or deterioration in each of the outcome parameters was attributed when a parameter showed a change of $>20 \%$ change in either direction after treatment. No change was attributed to a parameter when the change after treatment was $\leqslant 20 \%$ compared with the baseline level. In patients withdrawn from the study the clinical response was evaluated by comparing baseline data with those obtained at the last visit for intent-to-treat analysis.

\section{Statistical methods and data analysis}

The objective of this trial was to evaluate the clinical efficacy of ICS treatment in bronchiectasis. The primary outcome variables of interest were 24 hour sputum volume and exacerbation frequency. There are no published data on the effects of ICS treatment on exacerbation frequency. Our follow up on 82 stable patients with bronchiectasis showed that $8 \%$ of patients would experience a spontaneous improvement in 24 hour sputum volume (unpublished data), defined as $>20 \%$ reduction in sputum volume, compared with baseline. We anticipated that treatment with fluticasone would increase the proportion of patients who improved to about $30 \%$, and power calculation showed that a sample size of 40 per group would have $80 \%$ power at a $5 \%$ alpha level. ${ }^{15}$

Data are expressed as mean (SD) unless otherwise stated. A logistic regression model was constructed on the subgroup of patients with a 24 hour sputum volume of $<30 \mathrm{ml}$ using sputum volume response as the dependent variable and exacerbation frequency, sputum purulence, sputum PA status, $\mathrm{FEV}_{1}$ and FVC as independent variables. Likewise, regression models were constructed on each of the subgroups of patients with exacerbation frequency of $\leqslant 2$ year and a sputum purulence score of $>5$ using the aforementioned parameters as independent variables where appropriate. Regression analysis was not undertaken for PA status in view of the small sample size.
Categorical variables, including clinical response in the primary and secondary outcome parameters, were compared between treatment groups using $\chi^{2}$ or Fisher's exact tests which also provided odds ratios (OR) and 95\% confidence intervals (CI). Continuous data were compared using the $t$ test or Wilcoxon rank sum test where appropriate. Unless otherwise stated, only intent-to-treat analysis was performed for outcome parameters for all the randomised patients. The analysis was performed using SPSS Version 10.0 (SPSS Inc, Chicago, IL, USA). A p value of $<0.05$ was considered statistically significant.

\section{RESULTS}

\section{Patient demography and clinical details}

Between September 1998 and May 1999, 86 patients were recruited to the study (table 1). There was no significant difference in sex distribution, age, age at onset of symptoms, smoking history, past medical history, current medications, sputum pathogens, or the number of bronchiectatic lung segments between the two groups $(\mathrm{p}>0.05)$. The aetiology of bronchiectasis did not differ between the two groups $(\mathrm{p}>0.05)$ and was as follows: idiopathic (fluticasone $\mathrm{n}=$ 35, placebo $n=34)$, post-tuberculous $(n=6, n=6)$, postpneumonic $(\mathrm{n}=0, \mathrm{n}=2)$, primary ciliary dyskinesia $(\mathrm{n}=2$, $\mathrm{n}=0)$, and IgG deficiency $(\mathrm{n}=0, \mathrm{n}=1)$. Sore throat was the only reported adverse reaction and affected seven (16.3\%) and two $(4.7 \%)$ patients in the fluticasone and placebo groups, respectively $(\mathrm{p}=0.16)$. Compliance (total dose taken by each patient at the end of treatment) was 90.6 (16.5)\% for the fluticasone group and 91.2 (12.4)\% for the placebo group $(\mathrm{p}=0.86)$

\section{Patient withdrawals}

The numbers of patients who completed the 52 week assessment in the fluticasone and placebo groups were 35 and 38 , respectively (fig $\mathrm{l}, \mathrm{p}=0.37$ ). In the fluticasone group eight patients were withdrawn for the following reasons:

Table 1 Clinical characteristics of patients in the fluticasone and placebo groups at baseline

\begin{tabular}{|c|c|c|}
\hline & $\begin{array}{l}\text { Fluticasone } \\
\text { ( } n=43,23 F \text { ) }\end{array}$ & $\begin{array}{l}\text { Placebo } \\
(n=43,34 F)\end{array}$ \\
\hline Age (years) & $57.7(14.4)$ & $59.2(14.2)$ \\
\hline Age at onset of symptoms (years) & $41.8(18.8)$ & $40.8(18.5)$ \\
\hline \multicolumn{3}{|l|}{ Smoking history } \\
\hline Never & 33 & 36 \\
\hline Ex & 8 & 7 \\
\hline Current & 2 & 0 \\
\hline \multicolumn{3}{|l|}{ Past medical history } \\
\hline Nil else & 26 & 25 \\
\hline Hypertension & 5 & 4 \\
\hline Hypothyroidism & 0 & 2 \\
\hline Pulmonary TB & 3 & 0 \\
\hline Ischaemic heart disease & 2 & 4 \\
\hline Peptic ulcer disease & 2 & 2 \\
\hline Others & 8 & 7 \\
\hline \multicolumn{3}{|l|}{ Current medications } \\
\hline None & 17 & 11 \\
\hline Inhaled bronchodilators & 11 & 19 \\
\hline Antihypertensives & 5 & 5 \\
\hline Theophylline or oral $\beta_{2}$ agonist & 7 & 7 \\
\hline Others & 12 & 8 \\
\hline \multicolumn{3}{|l|}{ Sputum pathogen } \\
\hline Commensals & 25 & 27 \\
\hline$P$ aeruginosa & 12 & 11 \\
\hline$H$ influenzae & 6 & 5 \\
\hline $\begin{array}{l}\text { No. of bronchiectatic lung } \\
\text { segments }\end{array}$ & $3.0(1.6)$ & $2.8(1.6)$ \\
\hline \multicolumn{3}{|c|}{$\begin{array}{l}\text { Data shown are mean (SD). } \\
\text { *No significant difference between fluticasone and placebo groups } \\
\text { ( } p>0.05 \text { ). }\end{array}$} \\
\hline
\end{tabular}




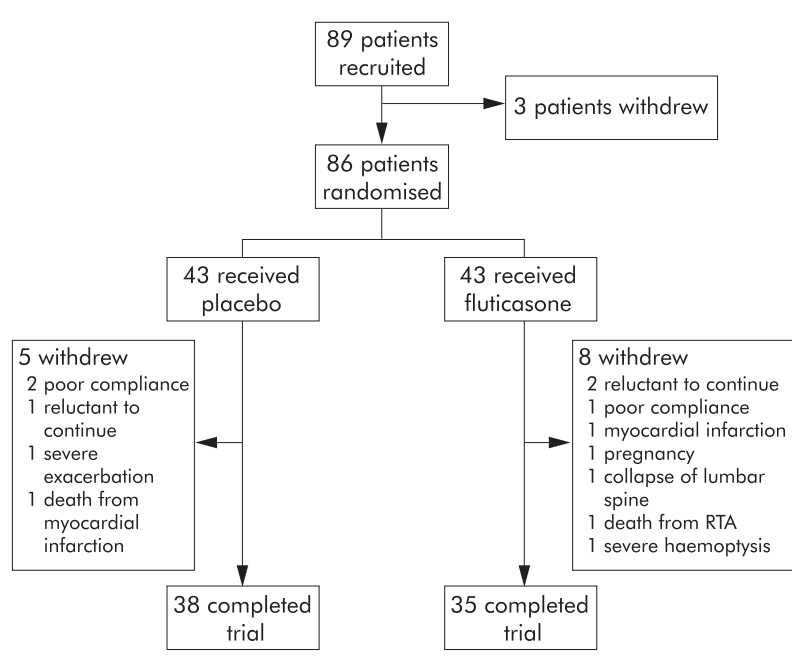

Figure 1 Trial profile.

poor compliance $(<50 \%$ of prescribed doses taken, $\mathrm{n}=1, \mathrm{M}$, 4 weeks), myocardial infarction $(\mathrm{n}=1, \mathrm{M}, 6$ weeks), pregnancy $(\mathrm{n}=1, \mathrm{~F}, 36$ weeks), reluctance to continue $(\mathrm{n}=2,2 \mathrm{~F}$, 4 and 22 weeks), death from road traffic accident $(n=1, F$, 52 weeks), severe haemoptysis ( $\mathrm{n}=1, \mathrm{~F}, 50$ weeks), and severe back pain due to collapse of fourth lumbar spine $(\mathrm{n}=1, \mathrm{~F}, 32$ weeks $)$. In the placebo group five patients were withdrawn as follows: reluctance to continue $(\mathrm{n}=1, \mathrm{~F}$, 4 weeks), poor compliance ( $\mathrm{n}=2, \mathrm{lM}, \mathrm{lF}$, both at 24 weeks), development of a severe exacerbation $(\mathrm{n}=1, \mathrm{~F}, 24$ weeks), and death from myocardial infarction $(n=1, M, 52$ weeks).

\section{4 hour sputum volume}

Logistic regression models undertaken with treatment modality, baseline sputum purulence score, baseline exacerbation frequency, sputum PA status, baseline $\mathrm{FEV}_{1}$, and baseline FVC as covariates and response of sputum volume as the dependent variable showed that fluticasone treatment had a significant beneficial effect on sputum volume compared with placebo in subgroups of patients with 24 hour sputum volume $<30 \mathrm{ml}(\mathrm{p}=0.04)$, exacerbation frequency $\leqslant 2 /$ year $\quad(p=0.04)$, and sputum purulence score $>5$ $(\mathrm{p}=0.03$; tables 2 and 3$)$. Of the patients with baseline 24 hour sputum volume $<30 \mathrm{ml}, 23$ showed improvement and 13 showed no improvement (nine showed deterioration, four no change) in post-treatment 24 hour sputum volume, which was significantly different from controls (16 showed improvement, 24 no improvement ( 10 showed deterioration, 14 no change), $p=0.04$, OR $2.7,95 \%$ CI 1.0 to 6.7 ). Thirteen and three patients with baseline exacerbation frequency $\leqslant 2$ year showed improvement and no improvement (one deterioration and two no change) in post-treatment 24 hour sputum volume, which was significantly different from the control group (11 improvement, 13 no improvement (three deterioration, 10 no change), $\mathrm{p}=0.047$, OR $5.1,95 \%$ CI 1.2 to 22.7). Of the 36 patients in the fluticasone group who had a sputum purulence score of $>5,23$ and 13 showed improvement and no improvement, respectively, in the post-treatment 24 hour sputum volume (four showed deterioration, nine no change) compared with the placebo group $(\mathrm{n}=35)$ where 14 showed improvement, 21 no improvement ( 10 showed deterioration, 11 no change), $p=0.06$, OR 2.7 , 95\% CI 1.0 to 6.9). However, this baseline sputum characteristic did not predict clinical outcome in post-treatment exacerbation frequency $(p=0.19$, data not shown). There was no significant difference in the post-treatment 24 hour sputum volume between the treatment groups $(p=0.72)$.

\section{Exacerbation frequency}

There were 96 and 117 exacerbations in the fluticasone and placebo groups, respectively, which were treated with levofloxacin. Of these, four and five patients were admitted to hospital (once each) for physiotherapy and other treatment, although levofloxacin remained the only antibiotic therapy. There was no difference in the number of patients who showed improvement between the two treatment groups $(p=0.37)$, nor was there any difference between the two groups in the exacerbation frequency after treatment $(\mathrm{p}=0.41$, tables 2 and 3$)$.

\section{Secondary outcome parameters and bronchiectasis symptoms}

There was no significant difference in the clinical response for post-treatment sputum purulence or sputum purulence scores between the treatment groups $(\mathrm{p}>0.05)$, nor was there a significant difference in the number of patients showing improvement or otherwise in $\mathrm{FEV}_{1}$ or FVC following treatment ( $\mathrm{p}=1.00$ and 1.00, respectively; tables 2 and 3 ). Between group comparisons revealed no significant difference in the percentage of patients with bronchiectasis symptoms in the two treatment groups $(\mathrm{p}>0.05)$, except that significantly fewer patients treated with fluticasone complained of post-treatment cough $(\mathrm{p}=0.03$; table 3$)$.

Table 2 Clinical responses of primary and secondary outcome parameters compared with baseline data for 86 patients by intent-to-treat analysis

\begin{tabular}{|c|c|c|c|c|c|c|}
\hline & \multicolumn{3}{|c|}{ Fluticasone group ( $n=43,23 F)$} & \multicolumn{3}{|c|}{ Placebo group ( $n=43,34 F)$} \\
\hline & \multicolumn{3}{|c|}{ Clinical response after treatment } & \multicolumn{3}{|c|}{ Clinical response after treatment } \\
\hline & $\begin{array}{l}\text { Improvement } \\
\text { n (\%) }\end{array}$ & $\begin{array}{l}\text { Deterioration } \\
\text { n (\%) }\end{array}$ & $\begin{array}{l}\text { No change } \\
\text { n (\%) }\end{array}$ & $\begin{array}{l}\text { Improvement } \\
\text { n (\%) }\end{array}$ & $\begin{array}{l}\text { Deterioration } \\
\text { n (\%) }\end{array}$ & $\begin{array}{l}\text { No change } \\
\text { n (\%) }\end{array}$ \\
\hline \multicolumn{7}{|c|}{ Primary outcome parameters } \\
\hline $\begin{array}{l}24 \text { hour sputum volume } \\
(\mathrm{ml})^{*}\end{array}$ & $28(65.1)$ & $5(11.6)$ & $10(23.3)$ & $18(41.9)$ & $10(23.2)$ & $15(34.9)$ \\
\hline $\begin{array}{l}\text { Exacerbation frequency } \\
\text { (/year) }\end{array}$ & $22(51.2)$ & $7(16.2)$ & $14(32.6)$ & $16(37.2)$ & $11(25.6)$ & $16(37.2)$ \\
\hline \multicolumn{7}{|c|}{ Secondary outcome parameters } \\
\hline Sputum purulence score & $13(30.2)$ & $10(23.3)$ & $20(46.5)$ & $9(20.9)$ & $15(34.9)$ & $19(44.2)$ \\
\hline $\mathrm{FEV}_{1} \%$ predicted & $2(4.7)$ & $4(9.3)$ & 37 (86.0) & $3(7.0)$ & $3(7.0)$ & 37 (86.0) \\
\hline FVC $\%$ predicted & $4(9.3)$ & $3(7.0)$ & $36(83.7)$ & $4(9.3)$ & $1(2.3)$ & $38(88.4)$ \\
\hline
\end{tabular}

${ }^{*} \mathrm{p}<0.05$ when both treatment groups compared for the number of patients who showed improvement or otherwise (deterioration or no change) using $\chi^{2}$ or Fisher's exact tests. 
Table 3 Primary and secondary outcomes and bronchiectasis symptoms assessed at baseline and after treatment by intent-totreat analysis

\begin{tabular}{|c|c|c|c|c|c|c|}
\hline & \multicolumn{2}{|c|}{ Fluticasone group ( $n=43,23 F$ ) } & \multicolumn{2}{|c|}{ Placebo group ( $n=43,34 F$ ) } & \multirow[b]{2}{*}{ p value* } & \multirow[b]{2}{*}{ Mean $(95 \% \mathrm{Cl})$ difference } \\
\hline & Baseline & After treatment & Baseline & After treatment & & \\
\hline \multicolumn{7}{|l|}{ Primary outcome parameters } \\
\hline 24 hour sputum volume $(\mathrm{ml}) \dagger$ & $10(5-25)$ & $5(2-20)$ & $10(5-20)$ & $5(3-20)$ & 0.72 & $-1.0(-6.56$ to 4.56$)$ \\
\hline $\begin{array}{l}\text { Exacerbation frequency } \\
\text { (/year)t }\end{array}$ & $4(2-5)$ & $2(1-4)$ & $2(1-4)$ & $2(1-3)$ & 0.41 & $0.42(-0.59$ to 1.43$)$ \\
\hline \multicolumn{7}{|l|}{ Secondary outcome parameters } \\
\hline Sputum purulence score & $6.1(2.0)$ & $5.7(2.7)$ & $6.0(1.8)$ & $5.5(2.7)$ & 0.73 & $-0.21(-1.39$ to 0.97$)$ \\
\hline $\mathrm{FEV}_{1} \%$ predicted & $74.1(29.4)$ & $74.4(31.5)$ & $74.4(29.2)$ & $74.4(29.9)$ & 1.00 & $-0.02(-13.19$ to 13.14$)$ \\
\hline FVC $\%$ predicted & $83.1(23.6)$ & $83.1(23.4)$ & $85.0(22.6)$ & $85.9(23.3)$ & 0.58 & $2.81(-7.2$ to 12.82$)$ \\
\hline \multicolumn{7}{|l|}{$\%$ of patients with } \\
\hline Cough & 76.7 & 60.5 & 93.0 & 81.4 & 0.03 & \\
\hline Dyspnoea & 16.3 & 2.3 & 23.3 & 4.7 & 1.00 & \\
\hline Haemoptysis & 39.5 & 9.3 & 51.2 & 4.7 & 0.68 & \\
\hline Chest pain & 32.6 & 18.6 & 41.9 & 16.3 & 0.78 & \\
\hline Wheezing & 39.5 & 23.3 & 46.5 & 20.9 & 0.80 & \\
\hline Fatigue & 74.4 & 55.8 & 65.1 & 69.8 & 0.18 & \\
\hline
\end{tabular}

Unless otherwise stated, data are shown as mean (SD).

* $p$ value for between group comparison on post-treatment data.

†Median (interquartile range).

\section{Effects of Pseudomonas aeruginosa (PA) infection}

At baseline PA was isolated in the sputum from 12 and 11 patients in the fluticasone and placebo groups, respectively $(5$ and $10 \mathrm{~F} ; 54$ (15.9) and 52 (12.1) years; $\mathrm{p}=0.03$ and 0.76 respectively). These patients had no significant difference in age at onset of symptoms, past medical history, current medications, or number of lung lobes affected by bronchiectasis ( $p>0.05$, data not shown). Of these, three patients were withdrawn from each of the treatment groups before 52 weeks, as outlined above. Post-treatment exacerbation frequency improved in 10 patients, deteriorated in one, and remained unchanged in one patient in the fluticasone group, which was significantly different from the placebo group (three, six, and two, respectively; OR 13.3, 95\% CI 1.8 to 100.2, $\mathrm{p}=0.01)$. Post-treatment 24 hour sputum volume improved in nine patients, deteriorated in one patient, and remained unchanged in two patients in the fluticasone group, which was significantly different from the placebo group (two, six and three, respectively; OR 13.5, 95\% CI 1.8 to 100.2, $\mathrm{p}=0.01)$. Between group comparison showed no significant difference in the continuous data in 24 hour sputum volume, exacerbation frequency, $\mathrm{FEV}_{1}, \mathrm{FVC}$, or sputum purulence score between the two groups after treatment ( $\mathrm{p}>0.05$, data not shown).

\section{DISCUSSION}

This is the first double blind, placebo controlled, randomised study to evaluate the clinical efficacy of medium term ICS treatment in bronchiectasis. Compared with placebo, ICS treatment was associated with improvement in 24 hour sputum volume in significantly more patients but not exacerbation frequency, $\mathrm{FEV}_{1}, \mathrm{FVC}$, or sputum purulence score. Significantly more fluticasone patients with PA infection (known to be associated with increased morbidity in bronchiectasis) showed improvement in 24 hour sputum volume and exacerbation frequency compared with the placebo group. We have previously shown that a 24 hour sputum volume of $\geqslant 30 \mathrm{ml}$, a purulence score of $>5$, and exacerbation frequency of $\geqslant 3$ are markers of severe bronchiectasis. ${ }^{4}{ }^{16}$ These clinically meaningful subgroups were therefore selected for further analysis, despite the relatively small sample size. Our data showed that predictors for improvement in 24 hour sputum volume following treatment with ICS include baseline 24 hour sputum volume $<30 \mathrm{ml}$, exacerbation frequency $\leqslant 2 /$ year, and sputum purulence score $>5$. There was a considerable placebo effect symptomatically as both groups of patients reported improvement in respiratory symptoms, perhaps due to the highly supervised treatment protocol. As in the previous 6 week ICS study, we also found that treatment with fluticasone for 52 weeks was associated with improvement in cough. Other pretreatment clinical parameters such as spirometry, age, age at onset of symptoms, and clinical symptoms did not predict a favourable response to ICS treatment.

Despite the aforementioned improvement in clinical response, there was no significant difference in the posttreatment continuous data between the two treatment groups. Our primary aim was to determine whether or not ICS treatment would improve the response rate in bronchiectasis. There is a very large inter-individual variability in 24 hour sputum volume and disease activity parameters in patients with bronchiectasis, and our study was not powered to look at the effects of treatment on these parameters. In addition, our study was specifically powered to evaluate the efficacy of ICS on 24 hour sputum volume but not exacerbation frequency. There are no published data to permit estimation of a sample size to evaluate the latter, although the data from this trial could facilitate such estimation for future studies. We are also aware of the potential problems which could invalidate our assumptions made for sample size calculation, such as the wide variations in sputum volume even for a single individual. Although we did not perform bronchial challenge testing to exclude asthma, none of our patients showed significant reversibility in spirometric parameters, diurnal variation in PEFR, or typical asthma symptoms. ${ }^{13}$ The baseline exacerbation frequency was derived retrospectively and could not replace prospective data.

Although bronchiectasis is the final common path for a wide spectrum of diseases, there is an identifiable aetiology in $60-80 \%{ }^{17}{ }^{18}$ and intensive investigations only reveal aetiological factors with implications for prognosis or specific treatment in $15 \%$ of patients. ${ }^{18}$ Specific or disease modifying treatment is not available and this is reflected by the continued deterioration of patients with $\mathrm{CF}$ and non-CF bronchiectasis. Treatment with mucolytics, methylxanthines, hyperosmolar agents, or leukotriene receptor antagonists has no effect on bronchiectasis. ${ }^{19}$ Although low dose erythromycin treatment improves lung function and sputum production, possibly by downregulation of epithelial glycoconjugate release, its long term effects in bronchiectasis are unknown. ${ }^{20}$ 
Administration of rhDNase, despite its proven efficacy in CF, worsens exacerbation frequency and $\mathrm{FEV}_{1}$ in non-CF bronchiectasis. ${ }^{21}$

Although patients with non-CF bronchiectasis do not develop the "cepacia syndrome", infection with PA is associated with increased sputum production, poorer lung function, and slowing of respiratory ciliary beat in vivo. ${ }^{42}$ Treatment with a nebulised aminoglycoside improves sputum PA density, lung function, and rates of hospital admission in patients with $\mathrm{CF}^{23}$ and reduces sputum PA density and myeloperoxidase in those with non-CF bronchiectasis. ${ }^{24}$ However, even prolonged potent antibiotic therapy cannot eradicate PA or prevent disease progression in bronchiectasis. Our data show that ICS treatment improves disease activity (manifest as exacerbation frequency and 24 hour sputum volume) in patients with PA infection. The interaction between PA and the respiratory mucosa is poorly understood and the role of corticosteroids in this process is even less clear. The efficacy of ICS treatment in this subgroup of patients suggests that corticosteroids could beneficially alter the interaction between PA or its toxins and the bronchiectatic airways. The lack of effective treatment against chronic PA infection in the airways of these patients makes it imperative to evaluate the underlying mechanism(s).

Although asthma, COPD, and bronchiectasis are similar in symptoms, airway inflammation, and airflow obstruction, ICS treatment is only of proven clinical benefit in asthma where it improves lung function and exacerbation frequency. ${ }^{25}$ Such efficacy is unclear in COPD and previously unexplored in bronchiectasis. While ICS treatment does not halt the progression of COPD, it appears to benefit patients with a spirometric response to ICS, $\mathrm{FEV}_{1}<50 \%$, or frequent exacerbations. ${ }^{26-29}$ Treatment with ICS also improves respiratory symptoms, frequency of physician visits, and lower airway reactivity in patients with COPD. ${ }^{28}{ }^{29}$ Unlike asthma, in which eosinophils play a major role, patients with bronchiectasis and COPD have neutrophils as the most prominent cells in the airways. As in COPD, our data show that ICS treatment does not improve lung function in bronchiectasis, even with over $90 \%$ compliance and after 52 weeks.

Glucocorticoids act by binding to a specific receptor which, upon activation, regulates expression of proinflammatory transcription factors such as activator protein-1, nuclear factor of activated $\mathrm{T}$ cells, and nuclear factor- $\mathrm{\kappa B}$ in asthma. ${ }^{30}$ The mechanism(s) for the efficacy of ICS treatment in COPD are less clear and are unexplored in bronchiectasis, although treatment with ICS reduces sputum output of leucocytes, IL$1 \beta$, IL- 8 , and LTB4 in the latter. ${ }^{1}$ It is probable that the improvement in sputum volume-which reflects a dampening down of disease activity-could be the result of downregulation of airway proinflammatory mediators. This could lead to amelioration of leucocyte trafficking and therefore less neutrophilic infiltration and release of toxic products into the bronchiectatic airway. ${ }^{2}$ The lack of response in exacerbation frequency with ICS treatment suggests that the occurrence of exacerbations is more dependent on other factors such as infection than on inflammation, at least among patients not infected with PA. There appears therefore to be a need to combine antimicrobial and anti-inflammatory treatment in bronchiectasis.

\footnotetext{
Authors' affiliations

K W Tsang, K C Tan, J C Ho, J Mak, C Ko, C Yan, W K Lam, M ChanYeung, University Department of Medicine, Queen Mary Hospital, The University of Hong Kong, Hong Kong

P L Ho, G C Ooi, Department of Microbiology, Queen Mary Hospital, The University of Hong Kong, Hong Kong
}

G L Tipoe, Department of Anatomy, Queen Mary Hospital, The University of Hong Kong, Hong Kong

This study was partially sponsored by GlaxoWelcome (Hong Kong).

\section{REFERENCES}

1 Tsang KW, Ho PL, Lam WK, et al. Inhaled fluticasone reduces sputum inflammatory indices in severe bronchiectasis. Am J Respir Crit Care Med 1998; 158:723-7.

2 Tsang KW, Chan K, Ho P, et al. Sputum elastase in steady-state bronchiectasis. Chest 2000;117:420-6.

3 Cole PJ. Inflammation: a two edged-sword - the model of bronchiectasis. Eur J Respir Dis 1986;147(Suppl):6-15.

4 Ho PL, Chan KN, Ip MS, et al. The effect of Pseudomonas aeruginosa infection on clinical parameters in steady-state bronchiectasis. Chest 1998;114:1594-8.

5 Amitani R, Wilson R, Rutman A, et al. Effects of human neutrophil elastase and bacterial proteinases on human respiratory epithelium. Am J Respir Cell Mol Biol 1991;4:26-32.

6 Morehead RS. Bronchiectasis in bone marrow transplantation. Thorax 1997;52:392-3.

7 Auerbach HS, Williams M, Kirkpatrick JA, et al. Alternate-day prednisolone reduces morbidity and improves pulmonary function in cystic fibrosis. Lancet 1985;2:686-8.

8 Elborn JS, Johnston B, Allen F, et al. Inhaled steroids in patients with bronchiectasis. Respir Med 1992:86:121-4.

9 Schiotz PO, Jorgensen M, Flensborg EW, et al. Chronic Pseudomonas aeruginosa in lung infection in cystic fibrosis. Acta Paediatr Scand 1983;72:283-7.

10 Van Haren EH, Lammers JW, Festen J, et al. The effects of inhaled corticosteroid budesonide on lung function and bronchial hyperresponsiveness in adult patients with cystic fibrosis. Respir Med 1995;89:209-14.

11 Nikolaizik WH, Schoni MH. Pilot study to assess the effect of inhaled corticosteroids on lung function in patients with cystic fibrosis. J Pediatr 1996; 128:271-4.

12 Balfour-Lynn IM, Klein NJ, Dinwiddie R. Randomised controlled trial of inhaled corticosteroids (fluticasone propionate) in cystic fibrosis. Arch Dis Child 1997:77:124-30.

13 American Thoracic Society. Evaluation of impairment/disability secondary to respiratory disorders. A statement of the American Thoracic Society. Am Rev Respir Dis 1986;133:205-9.

14 Naidich DP, McCauley DI, Khouri NF, et al. Computed tomography of bronchiectasis. J Comput Assist Tomogr 1982;6:437-44.

15 Altman DG, Gore SM. Statistics in practice. England: Devonshire Press, 1982.

16 Chan SL, Chan-Yeung M, Ooi GC, et al. Validation of the Hong Kong Chinese version of St George's Respiratory Questionnaire in patients with bronchiectasis. Chest 2002;122:2030-7.

17 Tsang KW, Lam SK, Lam WK, et al. High seroprevalence of Helicobacter pylori in active bronchiectasis. Am J Respir Crit Care Med 1998; 158:1047-51

18 Pasteur MC, Helliwell SM, Houghton SJ, et al. An investigation into causative factors in patients with bronchiectasis. Am J Respir Crit Care Med 2000;162:1277-84.

19 Barker AF. Bronchiectasis. N Engl J Med 2002;346:1383-93.

20 Tsang KW, Ho PL, Chan KN, et al. A pilot study of low-dose erythromycin in bronchiectasis. Eur Respir J 1999;13:361-4.

21 O'Donnell AE, Barker AF, llowite JS, et al. Treatment of idiopathic bronchiectasis with aerosolized recombinant human DNase I. rhDNase Study Group. Chest 1998;113:1329-34.

22 Tsang KW, Rutman A, Tanaka E, et al. Interaction of Pseudomonas aeruginosa with human respiratory mucosa in vitro. Eur Respir 1994;7:1746-53.

23 Ramsey BW, Pepe MS, Quan JM, et al. Intermittent administration of inhaled tobramycin in patients with cystic fibrosis. Cystic Fibrosis Inhaled Tobramycin Study Group. N Engl J Med 1999;340:23-30.

24 Lin HC, Cheng HF, Wang CH, et al. Inhaled gentamicin reduces airway neutrophil activity and mucus secretion in bronchiectasis. Am J Respir Crit Care Med 1997; 155:2024-9.

25 Van Essen-Zandvliet EE, Hughes MD, Waalkens $\mathrm{HJ}$, et al. Effects of 22 months of treatment with inhaled corticosteroids and/or beta2-agonists on lung function, airway responsiveness and symptoms in children with asthma. Am Rev Respir Dis 1992; 146:547-54.

26 Vestbo J, Sorensen T, Lange P, et al. Long-term effect of inhaled budesonide in mild and moderate chronic obstructive pulmonary disease: a randomised controlled trial. Lancet 1999;353:1819-23.

27 Pauwels RA, Lofdahl CG, Laitinen LA, et al. Long-term treatment with inhaled budesonide in persons with mild chronic obstructive pulmonary disease who continue smoking: European Respiratory Society Study on Chronic Obstructive Pulmonary Disease. NEngl J Med 1999;340:1948-53.

28 Burge PS, Calverley PM, Jones PW, et al. Randomised, double blind, placebo controlled study of fluticasone propionate in patients with moderate to severe chronic obstructive pulmonary disease: the ISOLDE trial. BMJ 2000;320: 1297-303

29 The Lung Health Study Research Group. Effect of inhaled triamcinolone on the decline in pulmonary function in chronic obstructive pulmonary disease. N Engl J Med 2000;343:1902-9.

30 Adcock IM. Glucocorticoid-regulated transcription factors. Pulm Pharmacol Ther $2001 ; 14: 211-9$. 